\title{
A CONGRUENCE FOR THE NUMBER OF ALTERNATING PERMUTATIONS
}

\author{
SUMIT KUMAR JHA
}

\begin{abstract}
Авstract. We present a new proof of a result of Knuth and Buckholtz concerning the period of the number of alternating congruences modulo an odd prime. The proof is based on properties of special functions, specifically the polylogarithm, Dirichlet eta and beta functions, and Stirling numbers of the second kind.
\end{abstract}

\section{INTRODUCTION}

Definition 1.1. Let $S_{n}$ be the set of all permutations of $\{1,2, \cdots, n\}$. A permutation $a_{1} a_{2} \cdots a_{n} \in S_{n}$ is called alternating if $a_{1}>a_{2}<a_{3}>a_{4}<\cdots$, that is, we have $a_{i}<a_{i+1}$ for $i$ even, and $a_{i}>a_{i+1}$ for $i$ odd. Let $A_{n}$ denote the number of alternating permutations in $S_{n}$.

The sequence $\left\{A_{n}\right\}$ has been studied by many authors and is listed as sequence Aooo111 [11] in the On-Line Encyclopedia of Integer Sequences. The first few values of $A_{n}$ are $1,1,2,5,16,61,272,1385,7936,50521, \cdots$.

The odd indexed terms of the sequence $\left\{A_{n}\right\}$ are the tangent numbers [12], and the even indexed terms are the secant numbers [13]. Knuth and Buckholtz [6, Theorem 1, 2 and 3] have studied the periodicity of these two sequences and obtained the same results which are in this paper, with the exception of the half-period result. Kummer $[8$, p. 372] obtained the periodicity and half-period results for the secant numbers. He did not discuss the congruence for tangent numbers, but his methods would work for the tangent numbers also.

DOI: 10.35834/YYYY/VVNNPPP

MSC2020: 05A05, 11B68, 11B73

Key words and phrases: Alternating permutations, Stirling numbers of the second kind, polylogarithm function 
S. K. JHA

\section{MAIN RESUlt}

Our main result is the following theorem.

Theorem 2.1. For every odd prime $p$ and positive integers $n, l$

$$
A_{l(p-1)+n} \equiv(-1)^{l\left(\frac{p-1}{2}\right)} A_{n} \quad(\bmod p) .
$$

To prove this theorem we first revisit some known related results.

\section{SOME KNOWN RESULTS}

The exponential generating function for the number of alternating permutations is given by

Theorem 3.1 ([9]). We have

$$
\sec z+\tan z=\sum_{n=0}^{\infty} \frac{A_{n} z^{n}}{n !} .
$$

Using the expansions for $\tan z$ and $\sec z$ in terms of the Bernoulli numbers $B_{r}$ and the Euler numbers $E_{r}$, respectively [5, equation (2.3) and equation (2.7)], we can conclude that

$$
A_{r}=\left\{\begin{array}{ll}
(-1)^{\frac{r-1}{2}} \frac{2^{r+1}\left(2^{r+1}-1\right) B_{r+1}}{r+1} & \text { when } r \text { is odd } \\
(-1)^{\frac{r}{2}} E_{r} & \text { when } r \text { is even }
\end{array} .\right.
$$

Definition 3.2. The polylogarithm function $\operatorname{Li}_{s}(z)$ is defined by the following power series in $z$

$$
\mathrm{Li}_{s}(z)=\sum_{k=1}^{\infty} \frac{z^{k}}{k^{s}}
$$

where $|z|<1$, or $|z|=1$ with $\Re(s)>1$, where $\Re(s)$ denotes the real part of $s$.

The polylogarithm function is a special case of the Lerch transcendent $\Phi(\lambda, s, a)$ defined by

$$
\Phi(\lambda, s, a)=\sum_{n=0}^{\infty} \frac{\lambda^{n}}{(n+a)^{s}}
$$

for any real $a>0$, and complex $\lambda$ and $s$ with either $\lambda<1$ for all $s \in \mathbb{C}$ or $|\lambda|=1$ with $\Re(s)>1$. Thus we have

$$
\operatorname{Li}_{s}(\lambda)=\lambda \Phi(\lambda, s, 1) .
$$

The series (3.3) extends to a function $\Phi(\lambda, s, a)$ which is analytic in $\lambda$ and $s$, for $\lambda \in$ $\mathbb{C} \backslash[1, \infty)$ and for all complex $s[4$, Lemma 2.2].

For the negative integer values of $s$, the Lerch transcendent function is given in terms of the Apostol-Bernoulli polynomials $\beta_{n}(a, \lambda)$ [1, equation (6.5)] as

$$
\Phi(\lambda,-r, a)=\frac{-\beta_{r+1}(a, \lambda)}{r+1},
$$


where the Apostol-Bernoulli polynomials can be defined by

$$
\frac{z e^{a z}}{\lambda e^{z}-1}=\sum_{n=0}^{\infty} \beta_{n}(a, \lambda) \frac{z^{n}}{n !} \quad(|z+\log \lambda|<2 \pi) .
$$

Thus we have

$$
\operatorname{Li}_{-r}(\lambda)=(-\lambda) \frac{\beta_{r+1}(a, \lambda)}{r+1} .
$$

It is also known that [2, formula 3.7]

$$
\mathrm{Li}_{-r}(z)=\left(z \frac{\partial}{\partial z}\right)^{r} \frac{z}{1-z}=\sum_{k=1}^{r} k ! S(r, k)\left(\frac{1}{1-z}\right)^{k+1} z^{k}
$$

where $S(r, k)$ are Stirling numbers of the second kind which can be defined by the recurrence $S(r+1, k+1)=(k+1) S(r, k+1)+S(r, k)$ with $S(n, 1)=1$ and $S(1, r)=0$ for $r>1$.

We also require the following fact from [3, p. 937]:

Lemma 3.3. We have, for an odd prime $p$,

$$
S(n+l(p-1), k) \equiv S(n, k) \quad(\bmod p)
$$

with integers $1 \leq k \leq p-1, n \geq 1$, and $l \geq 1$.

\section{Two Formulas for $A_{r}$}

Lemma 4.1. Let $i=\sqrt{-1}$. We have

$$
A_{r}=2 i^{r+1} \mathrm{Li}_{-r}(-i)
$$

where $\mathrm{Li}_{-r}(\cdot)$ is negative polylogarithm function.

Proof. Following equation (3.5) we have

$$
\mathrm{Li}_{-r}(-i)=i \frac{\beta_{r+1}(1,-i)}{r+1} .
$$

Let $\left[z^{n}\right] f(z)$ denote the the coefficient of $\frac{z^{n}}{n !}$ in the Taylor series expansion of $f(z)$ within the circle of convergence. Then

$$
\begin{aligned}
\beta_{r}(1,-i)=\left[z^{r}\right]\left(\frac{z e^{z}}{e^{z}(-i)-1}\right) & =\left[z^{r}\right](i)\left(\frac{z}{2}(\tanh z+1)+\frac{i z}{2} \operatorname{sech} z\right) \\
& =i\left(2^{r-1}\left(2^{r}-1\right) B_{r}+\frac{i r}{2} E_{r-1}\right),
\end{aligned}
$$

where in the last step we have used the expansions of $\tanh z$ and $\operatorname{sech} z$ in terms of the Bernoulli numbers $B_{r}$ and the Euler numbers $E_{r}$, respectively [5, equation (2.3) and equation (2.7)]. The above result allows us to conclude that

$$
\mathrm{Li}_{-r}(-i)=-\left(2^{r^{2}} \frac{r^{r+1}-1}{r+1} B_{r+1}+i \frac{E_{r}}{2}\right) \text {. }
$$


S. K. JHA

As $B_{r+1}=0$ when $r$ is even and $E_{r}=0$ when $r$ is odd, an application of equation (3.1) assures the validity of equation (4.1).

Corollary 4.2. Let $i=\sqrt{-1}$. We have

$$
A_{r}=i^{r} \sum_{k=1}^{r} \frac{(-1)^{k} k ! S(r, k)}{2^{k}}(i+1)^{k+1} .
$$

Proof. We substitute $z=-i$ in the above equation (3.6), multiply by $2 i^{r+1}$ and simplify to conclude equation (4.2).

\section{Proof of Theorem 2.1}

Proof of Theorem 2.1. Letting $r=l(p-1)+n$, where $p$ is an odd prime, in equation (4.2) we get

$$
\begin{aligned}
A_{l(p-1)+n} & =i^{l(p-1)+n} \sum_{k=1}^{p-1} \frac{(-1)^{k} k ! S(l(p-1)+n, k)}{2^{\frac{k-1}{2}}}\left(\frac{i+1}{\sqrt{2}}\right)^{k+1} \\
& +i^{l(p-1)+n} \sum_{k=p}^{l(p-1)+n} \frac{(-1)^{k} k ! S(l(p-1)+n, k)}{2^{\frac{k-1}{2}}}\left(\frac{i+1}{\sqrt{2}}\right)^{k+1} .
\end{aligned}
$$

The first term of the right hand side in the above equation is clearly congruent to $(-1)^{l\left(\frac{p-1}{2}\right)} A_{n}(\bmod p)$ in the light of Lemmas 4.2 and 3.3 .

Let $p \leq k \leq l(p-1)+n$. It is clear that $k !$ is a multiple of $p$ and that the power of 2 in the prime factorization of $k !$ is at least $[k / 2]([x]$ denotes the greatest integer $\leq x)$. Since $p$ is an odd prime, the power of 2 in the prime factorization of the integer $k ! / p$ is still at least $[k / 2]$. Thus when $k$ is odd $k ! / 2^{(k-1) / 2}$ is an integer which is a multiple of $p$, and the term $((i+1) / \sqrt{2})^{k+1}=e^{\frac{i \pi(k+1)}{4}}$ is a complex number with both real and imaginary parts being integers.

Suppose $k$ is even then $k ! / 2^{k / 2}$ is an integer which is a multiple of $p$, and the term

$$
\sqrt{2}\left(\frac{i+1}{\sqrt{2}}\right)^{k+1}=\sqrt{2} e^{\frac{i \pi(k+1)}{4}}
$$

is a complex number with both real and imaginary parts being integers.

This allows us to conclude that the second term in the right side of the equation (5.1) is a multiple of $p$, and hence our result equation (2.1).

\section{RELATEd RESUlts}

Remark I (Zhang's congruence). Zhang [10] proved that $A_{p-1} \equiv-1+(-1)^{\frac{p-1}{2}}(\bmod p)$ for all odd primes $p$. This congruence can also be proved by our method. We have

$$
A_{p-1}=(-1)^{\frac{p-1}{2}} \sum_{k=1}^{p-1} \frac{(-1)^{k} k ! S(p-1, k)}{2^{k}}(i+1)^{k+1} .
$$


Using the explicit form for the Stirling numbers of the second kind [3] we have

$$
(-1)^{k} k ! S(p-1, k)=\sum_{j=1}^{k}(-1)^{j}\left(\begin{array}{l}
k \\
j
\end{array}\right) j^{p-1} \equiv \sum_{j=1}^{k}(-1)^{j}\left(\begin{array}{l}
k \\
j
\end{array}\right) \equiv-1 \quad(\bmod p)
$$

for all $1 \leq k \leq p-1$. Thus operating in the field $\mathbb{Z} / p \mathbb{Z}$ we see that

$$
\begin{aligned}
2^{p-1} A_{p-1} & =(-1)^{\frac{p+1}{2}} \sum_{k=1}^{p-1}(i+1)^{k+1} 2^{p-1-k} \\
& =(-1)^{\frac{p+1}{2}} 2^{p-1}\left((i-1)-2 i 2^{-\frac{p}{2}} e^{\frac{i \pi p}{4}}\right) .
\end{aligned}
$$

Discarding the imaginary part in above we have

$$
A_{p-1} \equiv(-1)^{\frac{p+1}{2}}\left(2^{\frac{p-1}{2}} \sqrt{2} \sin (p \pi / 4)-1\right) \quad(\bmod p)
$$

Noting that $2^{\frac{p-1}{2}} \equiv(-1)^{\frac{p^{2}-1}{8}}(\bmod p)[7, \mathrm{p} .44]$ and $\sqrt{2} \sin (p \pi / 4)=(-1)^{\frac{-(p-1)(p-3)}{8}}$, which completes our alternative proof of Zhang's congruence.

Remark 2 (Generalization). Theorem 2.1 can be generalized using Euler's generalization for Fermat's little theorem as: Let $N$ be any natural number. Suppose $n$ and $l$ are positive integers with $n \geq N$. Then we have

$$
A_{l \cdot \phi\left(p^{N}\right)+n} \equiv(-1)^{l \cdot \frac{\phi\left(p^{N}\right)}{2}} A_{n} \quad\left(\bmod p^{N}\right) .
$$

\section{ACKNOWLEDGEMENT}

The author would like to thank the editor (Allen Stenger) and two anonymyous reviewers for their careful and detailed reviews which has brought the paper to its present form.

\section{REFERENCES}

[1] K. N. Boyadzhiev, Apostol-Bernoulli functions, derivative polynomials and Eulerian polynomials, Advances and Applications in Discrete Mathematics 1 (2008), 109-122. Preprint available at https: //arxiv.org/abs/0710.1124.

[2] K. N. Boyadzhiev, A series transformation formula and related polynomials, Int. J. Math. Math. Sci., Article Id. 792107, 2005.

[3] K. Dilcher, An extension of Fermat's little theorem, and congruences for Stirling numbers, Amer. Math. Monthly 107 (2000), 936-940.

[4] J. Guillera and J. Sondow, Double integrals and infinite products for some classical constants via analytic continuations of Lerch's transcendent, Ramanujan J. 16 (2008), 247-270.

[5] A. Hodges and C. V. Sukumar, Bernoulli, Euler, permutations and quantum algebras, Proc. R. Soc. A. 463 (2007), 2401-2414.

[6] D.E. Knuth and T. J. Buckholtz, Computation of tangent, Euler, and Bernoulli numbers, Math. Comp. 21 (1967), 663-688.

[7] N. Koblitz, A Course in Number Theory and Cryptography, Graduate Texts in Math. No. 114, SpringerVerlag, New York, 1987. 
S. K. JHA

[8] E. E. Kummer, Über eine allgemeine Eigenschaft der rationalen Entwickelungscoëfficienten einer bestimmten Gattung analytischer Functionen, J. Reine Angew. Math. 41 (1851), 368-372.

[9] R. P. Stanley, A survey of alternating permutations, Contemp. Math. 531 (2010), 165-196.

[10] W. P. Zhang, Some identities involving the Euler and the central factorial numbers, Fibonacci Quart. 36 (1998), 154-157.

[11] OEIS Foundation Inc. (2020), The On-Line Encyclopedia of Integer Sequences, http://oeis.org/A000111.

[12] OEIS Foundation Inc. (2020), The On-Line Encyclopedia of Integer Sequences, http://oeis.org/A000182.

[13] OEIS Foundation Inc. (2020), The On-Line Encyclopedia of Integer Sequences, http://oeis.org/A000364.

INTERNATIONAL Institute of Information Technology, Hyderabad-500032, India

Email address: kumarjha.sumit@research.iiit.ac.in 